\title{
Complex parameterization of triangulations on oriented maps
}

\author{
Mathieu Dutour Sikirić * \\ Rudjer Bosković Institute, Bijenička 54, 10000 Zagreb, Croatia
}

Received 12 October 2011, accepted 9 April 2012, published online 1 June 2012

\begin{abstract}
We consider here triangulations of oriented maps having a specified set $S$ of vertices of degree different from 6 and some other vertices of degree 6 . Such map can be described by specifying the relative positions between elements of $S$ using Eisenstein integers. We first consider the case of 1 parameter, which corresponds to the Goldberg-Coxeter construction. Then we develop the general theory, the special case of positive curvature studied by Thurston and finally extend the theory to quadrangulations and some other cases. In the last section we expose application of parameterizations to the study of zigzags.
\end{abstract}

Keywords: Maps, graphs, Groups, parameterizations.

Math. Subj. Class.: 05C10, 57M20

\section{Triangulations of oriented maps}

By a $\left(\left\{v_{1}, \ldots, v_{m}\right\}, k\right)$-map we denote a map on an oriented surface with faces of size $k, v_{i}$ vertices of degree $i$ and "map" being "sphere" (genus $g=0)$, "torus" $(g=1)$ or "oriented map of genus $g$ ". For example, a $\left(\left\{v_{5}=2, v_{7}=2, v_{6}\right\}, 3\right)$-torus denotes a triangulation with 2 vertices of degree 5,7 and an unspecified number of vertices of degree 6 . We will be mostly concerned with the description of $(v, 3)$-maps of genus $g$. Euler relation for them reads as

$$
\sum_{j \geq 3} v_{j}(6-j)=12(1-g) .
$$

The dual of $\left(\left\{v_{5}=12, v_{6}\right\}, 3\right)$-spheres are 3-regular plane graphs, whose faces are 5 - or 6-gonal. Such graphs, named fullerenes, occur in chemistry [13] following the discovery of Buckminsterfullerene (also called truncated icosahedron, soccer ball) in 1985 [22].

\footnotetext{
*The author has been supported by the Croatian Ministry of Science, Education and Sport under contract 098-0982705-2707.

E-mail address: mdsikir@irb.hr (Mathieu Dutour Sikirić)
} 
Formula (1.1) can be interpreted as a Gauss-Bonnet formula and $6-j$ as the curvature of a vertex of degree $j$. A triangulation is said to be of positive curvature if all vertices have non-negative curvature. This implies that the possible vertex degrees belong to $\{3,4,5,6\}$ and the $v$-vector satisfies $3 v_{3}+2 v_{4}+v_{5}=12$ with $v_{6}$ unspecified. All 19 possibilities for $\left(v_{3}, v_{4}, v_{5}\right)$ are given below:

$\begin{array}{ccccccc}(0,0,12) & (0,1,10) & (0,2,8) & (0,3,6) & (0,4,4) & (0,5,2) & (0,6,0) \\ (1,0,9) & (1,1,7) & (1,2,5) & (1,3,3) & (1,4,1) & (2,0,6) & (2,1,4) \\ (2,2,2) & (2,3,0) & (3,0,3) & (3,1,1) & (4,0,0) & & \end{array}$

For the enumeration of 3-regular plane graphs with a specified face vector $\left(p_{i}\right)$, i.e. number $p_{i}$ of faces of size $i$ the program CPF by T. Harmuth [2] is very efficient. Another little known program by the same author is CGF [19], which can enumerate 3-regular oriented maps of specified genus and face vector. The corresponding program for 4-regular plane graphs is ENU by $\mathrm{O}$. Heidemeier [2]. All above mentioned programs are available from [1] and give by duality triangulations and quadrangulations.

The symmetry groups of fullerenes and other plane graphs of positive curvature were determined in $[13,5,12,7,8,6]$. For a given group $G$ of symmetry of a map, denote by $\operatorname{Rot}(G)$ the subgroup of index 1 or 2 of $G$ formed by the orientation preserving transformation. The class of a group $G$ is the set of groups $G^{\prime}$ having $\operatorname{Rot}\left(G^{\prime}\right)=G$. In Table 1 we give the possible groups of $(v, 3)$-spheres of positive curvature by their class $\operatorname{Rot}(G)$, where we used Schoenflies nomenclature for point groups. For any class the number of vertices of positive curvature is finite and the number of vertices of degree 6 is unspecified. Since there is essentially only one 6-regular plane triangulation, one sees that the positions of the vertices of positive curvature allow to define the map. We want to encode the positions by complex Eisenstein numbers $z \in \mathbb{Z}[\omega]$ with $\omega=e^{i \pi / 3}$.

In Section 2 we describe the simple cases of 1 or 2 parameters. The case of 1 parameter corresponds to the Goldberg-Coxeter construction [9]. In Section 3 we first explain the general theory of complex parameterization of $(v, 3)$-maps on oriented surfaces. Then we explain Thurston's theory [28] which gives stronger results for the case of spheres of positive curvature. Finally we explain the extension to $(v, 4)$-maps, self-dual spheres and $(v, 6)$-spheres.

Applications to zigzags are considered in Section 4. A very basic application of parameterization is for generating maps efficiently provided that the number of parameters is not too high. Another application considered in [11] is for eigenvalue estimation where it was proved that for any interval $[a, b] \subset[-3,3]$ there is a finite number of graphs of positive curvature having no eigenvalue in $I$.

We choose to emphasize Eisenstein parameter description but it is of course possible to consider descriptions by integral parameters. This is done in [16, 15] for fullerenes and this allows to write parameterizations for each group, not just rotation subgroup. Another real parameter descriptions, by so called dihedral angles, is developed in [26], but it is more suited for describing manifolds than graphs.

\section{One and two parameter constructions}

\subsection{The Goldberg-Coxeter construction}

The Goldberg-Coxeter construction takes a $(v, 3)$-map $\mathcal{M}$, two integers $k, l \geq 0$ and returns another $\left(\left\{v, v_{6}\right\}, 3\right)$-map $G C_{k, l}(\mathcal{M})$ of the same genus by adding triangles and vertices of 


$\left(\left\{v_{3}=4, v_{6}\right\}, 3\right)$-spheres
\begin{tabular}{|c|c|c|}
\hline class & all group & \# param \\
\hline$D_{2}$ & $D_{2}, D_{2 d}, D_{2 h}$ & 2 \\
$T$ & $T, T_{d}$ & 1 \\
\hline
\end{tabular}

\begin{tabular}{|c|c|c|}
\hline class & all group & \# param \\
\hline$C_{1}$ & $C_{1}, C_{s}, C_{i}$ & 4 \\
\hline$C_{2}$ & $C_{2}, C_{2 v}, C_{2 h}$ & 3 \\
\hline$D_{2}$ & $D_{2}, D_{2 d}, D_{2 h}$ & 2 \\
\hline$D_{3}$ & $D_{3}, D_{3 d}, D_{3 h}$ & 2 \\
\hline$D_{6}$ & $D_{6}, D_{6 h}$ & 1 \\
\hline$O$ & $O, O_{h}$ & 1 \\
\hline
\end{tabular}

\begin{tabular}{|c|c|c|}
\hline class & all group & \# param \\
\hline$C_{1}$ & $C_{1}, C_{s}, C_{i}$ & 10 \\
\hline$C_{2}$ & $C_{2}, C_{2 h}, C_{2 v}$ & 6 \\
\hline$C_{3}$ & $C_{3}, C_{3 h}, C_{3 v}$ & 4 \\
\hline$D_{2}$ & $D_{2}, D_{2 h}, D_{2 d}$ & 4 \\
\hline$D_{3}$ & $D_{3}, D_{3 h}, D_{3 d}$ & 3 \\
\hline$D_{5}$ & $D_{5}, D_{5 h}, D_{5 d}$ & 2 \\
\hline$D_{6}$ & $D_{6}, D_{6 h}, D_{6 d}$ & 2 \\
\hline$T$ & $T, T_{h}, T_{d}$ & 2 \\
\hline I & $I, I_{h}$ & 1 \\
\hline
\end{tabular}

Table 1: The classes of symmetry groups of $\left(\left\{v_{a}, v_{6}\right\}, 3\right)$-sphere.

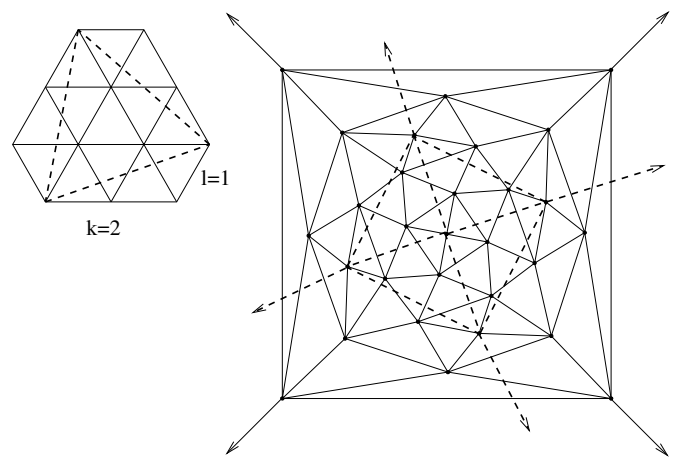

Figure 1: The construction of $G C_{2,1}($ Octahedron $)$.

degree 6 . It works by breaking the triangles of $\mathcal{M}$ into smaller triangles and gluing the pieces together in order to get another triangulation. See an example on Figure 1 and [9] for more details.

If $\mathcal{M}$ has $n_{T}$ triangles then $G C_{k, l}(\mathcal{M})$ has $n_{T}\left(k^{2}+k l+l^{2}\right)$ triangles. Since $k^{2}+k l+$ $l^{2}=|k+l \omega|^{2}$ it makes sense to associate the Eisenstein integer $z=k+l \omega$ to the pair $k, l$. The parameter symmetry $z \mapsto z \omega^{r}$ does not change the resulting map. All the cases of 1 parameter in Table 1 are described by the Goldberg-Coxeter construction of one plane graph. For example, if a $\left(\left\{v_{3}=4, v_{6}\right\}, 3\right)-,\left(\left\{v_{4}=6, v_{6}\right\}, 3\right)-,\left(\left\{v_{5}=12, v_{6}\right\}, 3\right)$-sphere is of symmetry $\left(I, I_{h}\right),\left(O, O_{h}\right)$ or $\left(T, T_{d}\right)$ then it is of the form $G C_{k, l}$ (Icosahedron), $G C_{k, l}$ (Octahedron) or $G C_{k, l}$ (Tetrahedron) [14,9]. Additionally, $\left(\left\{v_{4}=6, v_{6}\right\}, 3\right)$ spheres of symmetry $\left(D_{6}, D_{6 h}\right)$ are obtained as $G C_{k, l}\left(\right.$ Prism $\left._{6}^{*}\right)$.

\subsection{One case of 2 parameters: $\left(\left\{v_{5}=12, v_{6}\right\}, 3\right)$-spheres of symmetry $D_{5}$}

The 5 -fold axis of such a sphere has to pass through a vertex of degree 5 . There are 5 vertices of degree 5 around it; so, by 5 -fold symmetry, 1 complex parameter is needed to 

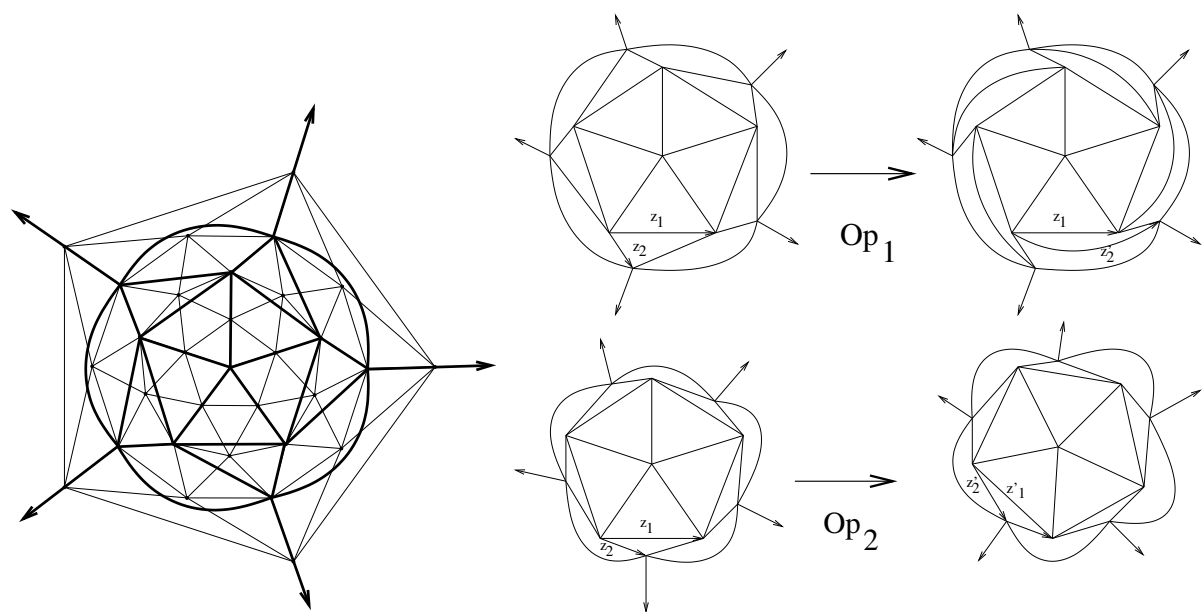

Figure 2: The parameterization of fullerenes of symmetry $D_{5}, D_{5 d}$ or $D_{5 h}$ in term of $\left(z_{1}, z_{2}\right) \in \mathbb{Z}[\omega]^{2}$ and two parameter operations.

describe them. Around those 5 vertices, there are 5 more vertices, so one more parameter is needed and then the last vertex is uniquely defined.

If one applies the following operations to parameters

- Operation 1: $\left(z_{1}, z_{2}\right) \mapsto\left(z_{1}, z_{1}+z_{2}\right)$

- Operation 2: $\left(z_{1}, z_{2}\right) \mapsto\left(z_{1}+\omega^{2} z_{2}, z_{1}-z_{2}\right)$

- Operation 3: $\left(z_{1}, z_{2}\right) \mapsto\left(z_{1}, z_{2}\right) \omega^{r}$

then one obtains the same sphere as a result. The group generated by those operations is named monodromy group. The number of triangles of $S$ is expressed as $q\left(z_{1}, z_{2}\right)=$ $10\left\{z_{1} \overline{z_{1}}+\frac{z_{1} \overline{z_{2}}-\overline{z_{1}} z_{2}}{\omega-\bar{\omega}}\right\}$. So, for a given pair $\left(z_{1}, z_{2}\right)$ a sphere may not exist, for example, if $q\left(z_{1}, z_{2}\right)<0$.

\subsection{Other two parameters descriptions}

Of course what has been done for fullerene of class $D_{5}$ applies just as well for fullerenes of class $D_{6}$ and similar simple description are possible for the remaining 2 parameter cases of Table 1 (See Figure 3 for two such cases).

For the $\left(\left\{v_{3}=4, v_{6}\right\}, 3\right)$-spheres a very explicit two parameter description is given in Figure 4. Geometrically this corresponds to the fact that any $\left(\left\{v_{3}=4, v_{6}\right\}, 3\right)$-sphere is obtained as the quotient of a $\left(\left\{v_{6}\right\}, 3\right)$-torus by a group of order 2 leaving invariant exactly 4 vertices. Clearly, the monodromy group is $\operatorname{PSL}(2, \mathbb{Z})$ and the number of triangles is expressed as $\frac{4}{\omega-\bar{\omega}}\left(z_{1} \overline{z_{2}}-\overline{z_{1}} z_{2}\right)$. This description was used in [21] to compute the eigenvalues of dual $\left(\left\{v_{3}=4, v_{6}\right\}, 3\right)$-spheres. 

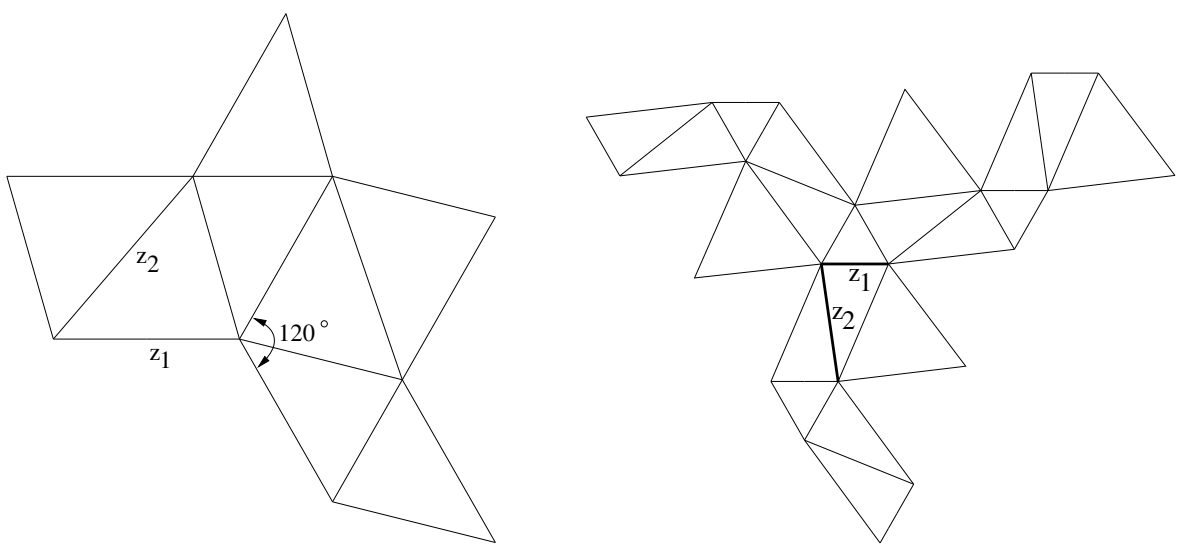

$\left(\left\{v_{4}=6, v_{6}\right\}, 3\right)$-spheres of symmetry $D_{2}, \quad\left(\left\{v_{5}=12, v_{6}\right\}, 3\right)$-spheres of symmetry $T$, $D_{2 h}, D_{2 d}$ $T_{h}$ and $T_{d}$

Figure 3: Two parameters description of two classes of spheres with positive curvature represented on the plane.

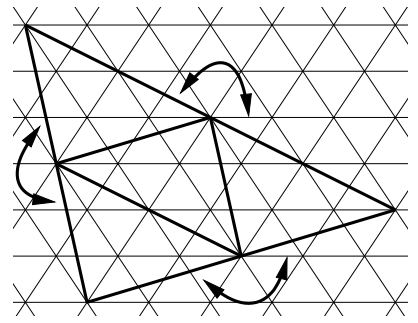

4 triangles in $\mathbb{Z}[\omega]$

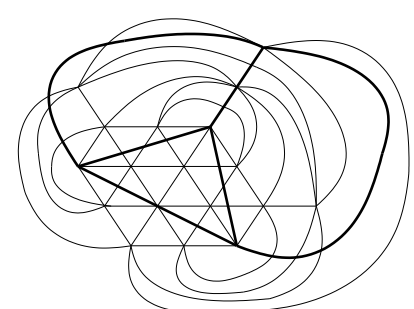

The triangulation

Figure 4: Description of $\left(\left\{v_{3}=4, v_{6}\right\}, 3\right)$-spheres. 


\section{Parameterization of maps on oriented surfaces}

\subsection{The general case}

The general Eberhard problem is for a given $g \geq 0$ and vector $\left(v_{i}\right)_{3 \leq i \leq m, i \neq 6}$ satisfying $\sum_{i=3}^{m}(6-i) v_{i}=12(1-g)$ to determine the set $P(v, g)$ of values of $v_{6}$ for which there exist a $\left(\left\{v, v_{6}\right\}, 3\right)$-oriented map of genus $g$. It is proved in [20] that $P(v, g)$ is empty only in the case $g=1$ and $v=\left\{v_{5}=1, v_{7}=1\right\}$ and the exact determination of $P(v, g)$ is an active subject of research.

Thus for a given $v$-vector it is interesting to consider how one can parametrize the $\left(\left\{v, v_{6}\right\}, 3\right)$-oriented maps of genus $g$. Let us call $\mathcal{M}$ such a map, $\widetilde{\mathcal{M}}$ its universal cover, $\Gamma$ its fundamental group and $S$ the set of vertices of degree different from 6 . By adding edges one by one, we can build a triangulation $T$ on $\mathcal{M}$ having $S$ as vertex set. Note that the degree of $v$ in $T$ is a priori not related to the degree of the corresponding vertex in $\mathcal{M}$. Naturally many triangulations are possible and they are mapped on the universal cover $\widetilde{\mathcal{M}}$ to $\Gamma$-invariant triangulations. We will see below that one can build a parameterization by Eisenstein integers from a triangulation.

Let us a take a triangulation $T$ and encode it combinatorially. A directed edge is an edge from a vertex to another vertex. Every edge $e$ is composed of a directed edge $\vec{e}$ and its reversal $r(\vec{e})$. The next operator $n$ maps a directed edge to the next one in clockwise order around the vertex $v$ in which it is contained. A triangulation $T$ is described by the operators $n$ and $r$ acting on the set of directed edges $D E(T)$. In particular the vertices, edges and faces of $T$ correspond to the orbits of $n, r$ and $n r$. For a given vertex $v$ of $T$ we denote by $\tilde{v}$ the corresponding vertex in the corresponding $\left(\left\{v, v_{6}\right\}, 3\right)$-map. Edges and faces of $T$ will have no direct analogs but homology classes will be mapped to homology classes.

We associate an Eisenstein integer $z_{\vec{e}} \in \mathbb{Z}[\omega]$ to any directed edge $\vec{e}$ of $T$. For any face $f=\left\{\overrightarrow{e_{1}}, \overrightarrow{e_{2}}, \overrightarrow{e_{3}}\right\}$ we impose the relation

$$
z_{\overrightarrow{e_{1}}}+z_{\overrightarrow{e_{2}}}+z_{\overrightarrow{e_{3}}}=0 .
$$

If we have an edge $e=\left\{\overrightarrow{e_{1}}, \overrightarrow{e_{2}}\right\}$ then we impose the consistency relation

$$
\omega^{\alpha\left(\overrightarrow{e_{1}}\right)} z \overrightarrow{e_{1}}+\omega^{\alpha\left(\overrightarrow{e_{2}}\right)} z_{\overrightarrow{e_{2}}}=0
$$

For any vertex $v$ containing the directed edges $\left(\vec{e}_{i}\right)_{1 \leq i \leq m}$ we have the relation

$$
6-\operatorname{deg}(\tilde{v}) \equiv \sum_{i=1}^{m} \alpha\left(\overrightarrow{e_{i}}\right)-\alpha\left(r\left(\overrightarrow{e_{i}}\right)\right) \quad(\bmod 6)
$$

with $\operatorname{deg}(\tilde{v})$ the degree of $\tilde{v}$. We write $D(v)=\operatorname{deg}(\tilde{v})$. If the triangulation $T$ is of genus $g$ then the first homology group $H_{1}(T)$ is $\mathbb{Z}^{2 g}$. For any cycle $C$ composed of directed edges $\left\{\overrightarrow{e_{1}}, \ldots, \overrightarrow{e_{m}}\right\}$ with $\vec{e}_{i+1}=r(n r)^{ \pm 1}\left(\vec{e}_{i}\right)$ we define the cycle sum

$$
I(C, \alpha)=\sum_{j} \alpha\left(\overrightarrow{e_{j}}\right)-\alpha\left(r\left(\overrightarrow{e_{j}}\right)\right)
$$

This sum depends on the chosen element of the homology class and defines how the orientation is shifted after one moves along $C$. If one adds 1 to $\alpha(\vec{e})$ and $\alpha(r(\vec{e}))$ then the 


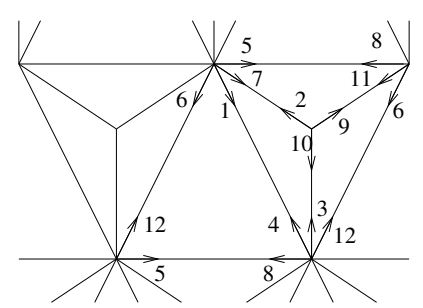

$\mathrm{A}\left(\left\{v_{3}=1, v_{9}=1, v_{6}\right\}, 3\right)$-torus

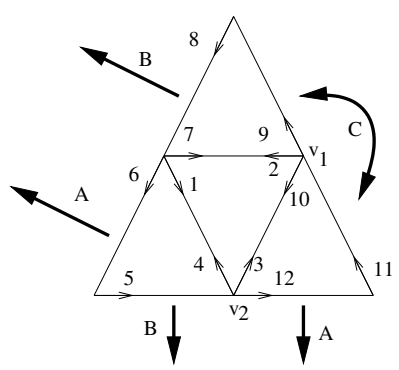

The parameterization

Figure 5: $\mathrm{A}\left(\left\{v_{3}=1, v_{9}=1, v_{6}\right\}, 3\right)$-torus and its parameterization. The identifications along $A, B$ and $C$ yields the equalities $\omega z_{12}+z_{6}=0, \omega z_{5}+z_{8}=0$ and $z_{9}-z_{11}=0$. We have $\operatorname{deg}\left(\tilde{v_{1}}\right)=3$ and $\operatorname{deg}\left(\tilde{v_{2}}\right)=9$.

resulting map $M(T, D, I, \alpha, z)$ does not change. The same happens if one adds 1 to $\alpha(\vec{e})$ for $\vec{e}$ in a face $F$ of the triangulation. In fact if we choose $2 g$ basic cycles $C_{i}$ of $T$ then any two vectors $\alpha, \alpha^{\prime}$ satisfying Equation $(3.1)$ and $I(C, \alpha) \equiv I\left(C, \alpha^{\prime}\right)(\bmod 6)$ differ by repeated application of above two operations. Equation (3.1) and the cycle sums $I$ allow to find a corresponding function $\alpha$ if it exists, which is not always the case. Henceforth the data of $T, D$ and $I$ determine the class of maps that one can obtain. In Figure 5 we give an example of a parameterization for $\left(\left\{v_{3}=1, v_{9}=1, v_{6}\right\}, 3\right)$-torus.

Theorem 3.1. The parameter spaces of $\left(\left\{v_{1}, \ldots, v_{m}\right\}, 3\right)$-oriented maps of genus $g$ have dimension $\sum_{3 \leq i \neq 6} v_{i}-1+2 g$ if all faces have size divisible by 6 and $\sum_{3 \leq i \neq 6} v_{i}-2+2 g$ otherwise.

Proof. Let us take such a map and build a triangulation $T$ on it. Let us write $M=$ $\sum_{3<i \neq 6} v_{i}$. We then construct a spanning tree of $M-1$ edges on the set of vertices of degree different from 6 . Since the map is of genus $g$ we have a basis of $2 g$ cycles of the group $H_{1}(G)$. We add $2 g$ edges to the spanning tree and the remaining edges define a tree in the dual map. Once we have defined the position of the $M-1+2 g$ edges, we have defined the triangulation uniquely because all other edges can be assigned iteratively. If one of the vertices has a degree not divisible by 6 then its position is defined uniquely once all its neighbors are known and so the dimension decreases by 1 in that case. No other relation exists since one can perturb the remaining parameter and still obtain some corresponding maps.

Let us call $m(v, g)$ the dimension in the above theorem.

For a given parameterization $(T, D, I)$ we denote by $q_{T}(z)$ the number of triangles of the obtained triangulation. The number of triangles contained in a face defined by $f=$ $\left\{\overrightarrow{e_{1}}, \overrightarrow{e_{2}}, \overrightarrow{e_{3}}\right\}$ is

$$
q_{f}(z)=\frac{1}{\omega-\bar{\omega}}\left(z_{\overrightarrow{e_{1}}} \overline{z_{\overrightarrow{e_{2}}}}-\overline{z_{\overrightarrow{e_{1}}}} z_{\overrightarrow{e_{2}}}\right)
$$

The function $q_{T}$ counting the total number of triangles is thus $q_{T}=\sum q_{f}$ and it is an Hermitian form. From this, one can deduce that for a fixed $v$ the number of $\left(\left\{v, v_{6}\right\}, 3\right)$ oriented maps of genus $g$ with at most $n$ triangles grows like $O\left(n^{m(v, g)}\right)$. Note that [27] gives the more precise estimate $O\left(n^{9}\right)$ for the number of fullerenes with exactly $n$ tri- 
angles. But we cannot have such a statement in the general case because for example $\left(\left\{v_{3}=4, v_{6}\right\}, 6\right)$-spheres exist only if $n$ is divisible by 4 .

Conjecture 3.2. If all vertices have degree divisible by 6 then the signature of $q_{T}$ is

$$
\left(n_{+}, n_{-}, n_{0}\right)=\left(g+\sum_{i} v_{i} F r\left(\frac{6-i}{6}\right), g, m(v, g)-n_{+}-n_{-}\right),
$$

otherwise, the signature is

$$
\left(n_{+}, n_{-}, n_{0}\right)=\left(g-1+\sum_{i} v_{i} F r\left(\frac{6-i}{6}\right), m(v, g)-n_{+}, 0\right)
$$

with $\operatorname{Fr}(x)=x-\lfloor x\rfloor$.

The conjecture is proved in the case $g=0$ in [24] and has been checked for the regular maps of genus at most 15 from [3].

For a given triangulation $T$ of a $\left(\left\{v, v_{6}\right\}, 3\right)$-oriented map, we choose $m$ independent parameters $z_{1}, \ldots, z_{m} \in \mathbb{Z}[\omega]$. The condition of existence of the map $M(T, D, I, \alpha, z)$ is that $q_{f}(z)>0$ for all face $f$ of $T$ and this defines the realizability space. The limit realizability space is the same space with $\mathbb{Z}[\omega]$ replaced by $\mathbb{C}$. The limit realizability space defines a set $S$ in the cone $q_{T}>0$. If one approach through a generic point of the boundary of $S$, which is not in the boundary of $q_{T}>0$ then one can rearrange the triangulation and get another triangulation $T^{\prime}$. The vertex degree $v$ in $T$ may change but the degree of $\tilde{v}$ remain the same. Similarly the cycles $C$ are mapped to cycles $C^{\prime}$ and $I\left(C^{\prime}, \alpha^{\prime}\right)=I(C, \alpha)$. Hence $D$ and $I$ are intrinsic to the class of triangulations obtained by rearrangements. Moreover, the parameter set $\left(z_{i}^{\prime}\right)$ of $\left(T^{\prime}, D, I, \alpha^{\prime}\right)$ can be expressed linearly in term of the parameter set $\left(z_{i}\right)$ of $(T, D, I, \alpha)$.

For two quadruples $(T, D, I, \alpha)$ and $\left(T^{\prime}, D, I, \alpha^{\prime}\right)$ of parameter set $\left(z_{i}\right),\left(z_{i}^{\prime}\right)$ we say that they are equivalent if there is a mapping from $T$ to $T^{\prime}$ preserving $D$ and $I$ such that $\left(z_{i}^{\prime}\right)$ can be expressed linearly from $\left(z_{i}\right)$. Such an equivalence preserve edge length, triangle areas and can be extended to adjacent triangulations of $T$ and correspondingly $T^{\prime}$. Note that some non-trivial equivalence can have $T=T^{\prime}$; this is the case for the 2 parameter description considered in Section 2 for which one triangulation suffices. The group of such transformation is the monodromy group and is a subgroup of $\mathrm{GL}_{m}(\mathbb{Z}[\omega])$ leaving invariant the form $q_{T}$. It may be that any two triples $(T, D, I),\left(T^{\prime}, D, I\right)$ are related by a sequence of such transformation but we have no proof of it and we do not know a counter-example.

\subsection{Thurston theory for maps of positive curvature}

Let us take one triple $\left(v_{3}, v_{4}, v_{5}\right)$ among the 19 triples of possible curvature. By Theorem 3.1 the number of complex parameters needed to describe $\left(\left\{v_{3}, v_{4}, v_{5}, v_{6}\right\}, 3\right)$-spheres is

$$
m=m\left(\left\{v_{3}, v_{4}, v_{5}\right\}, 0\right)=v_{3}+v_{4}+v_{5}-2
$$

The monodromy group is denoted by $M\left(\left\{v_{3}, v_{4}, v_{5}\right\}, 3,0\right)$ and it preserves the form $q$, which is of signature $(1, m-1,0)$. This class of monodromy groups was defined and enumerated in $[4,23,28]$ and in particular they are discrete groups. The 19 possible $\left(v_{3}, v_{4}, v_{5}\right)$ cases are part of the 94 cases determined there and the form $q$ is the intersection form on $H^{1}\left(S^{2}-V, L\right)$ with $V$ a set of $m+2$ points and $L$ a line bundle on $S^{2}-V$. 


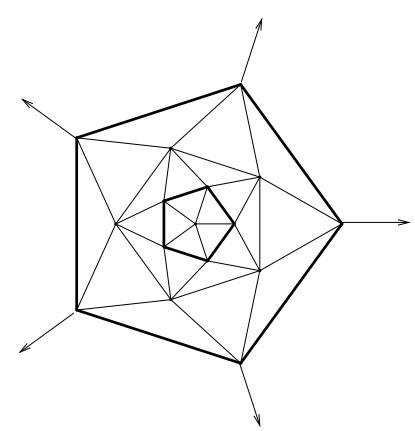

$30, D_{5 h}$

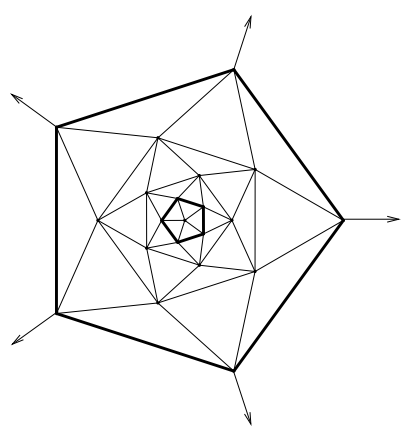

$40, D_{5 d}$

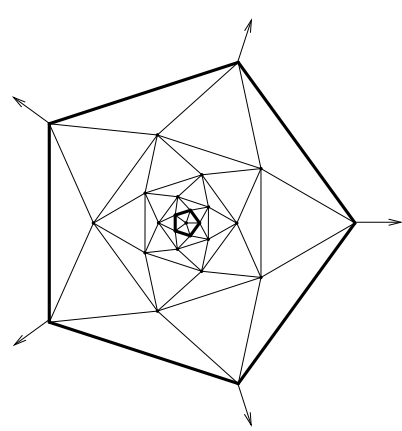

$50, D_{5 h}$

Figure 6: Three fullerenes of symmetry $D_{5}$ or more.
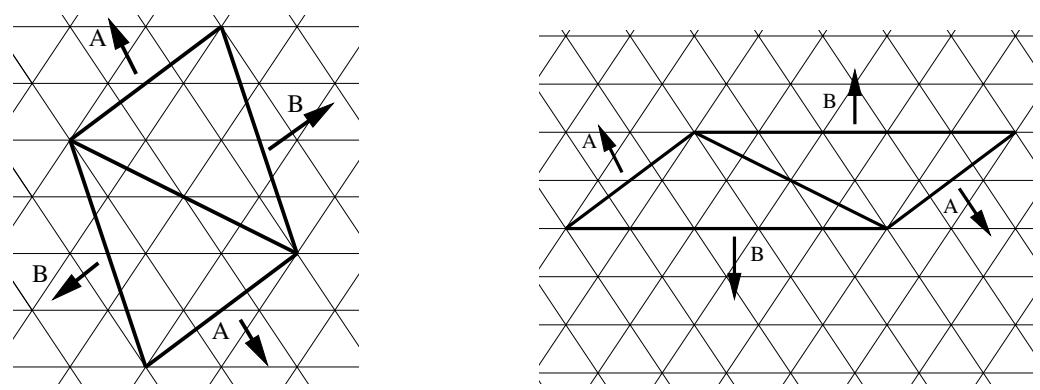

Figure 7: Two representations of a $(\{6\}, 3)$-torus.

In [28] it is proved that if $z \in \mathbb{Z}[\omega]^{m}$ and $q(z)>0$ then there exists $f \in M\left(\left\{v_{3}, v_{4}, v_{5}\right\}\right.$, $3,0)$ such that $f(z)$ is realizable as a $\left(\left\{v_{3}, v_{4}, v_{5}, v_{6}\right\}, 3\right)$-sphere. Thus $\mathbb{H}^{m} \cap \mathbb{Z}[\omega]^{m}$ up to the action of the monodromy group is a parameter space for the $\left(\left\{v_{3}, v_{4}, v_{5}, v_{6}\right\}, 3\right)$ spheres. As a consequence, the quotient

$$
\mathbb{H}^{m} /\left(\mathbb{R}_{>0} \times M_{3}\left(\left\{v_{3}, v_{4}, v_{5}\right\}, 3,0\right)\right)
$$

is of finite covolume because the number of $\left(\left\{v_{3}, v_{4}, v_{5}, v_{6}\right\}, 3\right)$-spheres is finite for any fixed number of triangles.

In [28] a characterization of the manifolds admitting a cocompact quotient is given. None of those corresponding to $\left(\left\{v_{3}, v_{4}, v_{5}, v_{6}\right\}, 3\right)$-spheres are compact. Each of the direction of non-compacity correspond to a partition of the vertices of non-zero curvature into two sets $S_{i}$ for $i=1,2$ each having $v_{q}^{i}$ vertices of degree $q$ and satisfying to $3 v_{3}^{i}+2 v_{4}^{i}+v_{5}^{i}=6$. Geometrically those are nanotubes, that is we have two caps $C_{i}$ with $\left(v_{3}^{i}, v_{4}^{i}, v_{5}^{i}\right)$ vertices separated by a number of rings of vertices of degree 6 (see Figure 6 ).

\subsection{Extensions and other cases of parameter descriptions}

One extension that can be done relatively simply is to consider vertices of degree 1 or 2 . For example, the $\left(\left\{v_{2}=3, v_{6}\right\}, 3\right)$-spheres are obtained by applying the Goldberg-Coxeter construction to the sphere reduced to a cycle of length 3 [18]. 
One example that does not fit exactly into the above scheme is the description of $\left(\left\{v_{6}\right\}, 3\right)$-torus by two parameters. It is done by writing down a parallelogram on the plane and identifying the sides. Since all vertices are equivalent in the torus, we have to choose one vertices of degree 6 used in the construction. In Figure 7 we show two equivalent representation of a $\left(\left\{v_{6}\right\}, 3\right)$-torus. One of the representation has one horizontal line in the fundamental domain; it is actually always possible to have such a representation and this is the basis of a 3 integral parameters construction [25].

Goldberg-Coxeter construction and complex parameterizations for $\left(\left\{v_{1}, v_{2}, v_{3}\right\}, 6\right)$ spheres are derived in [6]. The method was to apply the truncation operation to each vertex in order to get a $\left(\left\{v_{2}, v_{4}, v_{6}\right\}, 3\right)$-sphere for which existing theory could be applied.

Yet another extension is for quadrangulations. The theory extends without difficulty, we are still dealing with triples $(T, D, I)$ but the ring of Eisenstein integers is replaced by the ring of Gaussian integers. For example, for $\left(\left\{v_{3}=8, v_{4}\right\}, 4\right)$-sphere, the number of Gaussian integer parameters is 6 and the number for the classes $\left(O, O_{h}\right),\left(D_{4}, D_{4 d}, D_{4 h}\right)$, $\left(D_{3}, D_{3 d}, D_{3 h}\right),\left(D_{2}, D_{2 d}, D_{2 h}\right),\left(C_{2}, C_{2 h}, C_{2 v}\right),\left(C_{1}, C_{s}, C_{i}\right)$, respectively is $1,2,2,3$, 4 , and 6 . As a byproduct of this parameterization, we also get a method for parametrizing self-dual plane graphs with faces of size 3 or 4, see [10] for details.

Finally note that all of the above can be specialized to get parameter description of families of maps having a specific symmetry group $G$ provided that $G$ contains only orientation preserving mappings. This is because the symmetry conditions are translated into linear equalities in the parameters.

\section{Zigzag}

In an oriented map a zigzag is a circuit of edges such that two consecutive share a face and vertex but three do not share a face.

\subsection{The Goldberg-Coxeter case}

For a triangulation $\mathcal{M}$ we define in [9] a permutation group $\operatorname{Mov}(\mathcal{M})$ and two elements $L$ and $R$. If $\operatorname{gcd}(k, l)=1$ then the lengths of zigzags of $G C_{k, l}(\mathcal{M})$ is computed from the cycle structure of the element $L \odot_{k, l} R$ of $\operatorname{Mov}(\mathcal{M})$. This element satisfies the defining relations $L \odot_{1,0} R=L, L \odot_{0,1} R=R$ and

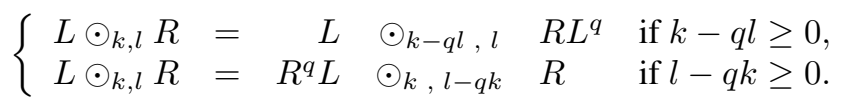

If $\operatorname{gcd}(k, l)=m>1$ then every zigzag of $G C_{k / m, l / m}(\mathcal{M})$ corresponds to $m$ zigzags of $G C_{k, l}(\mathcal{M})$ of length multiplied by $m$. The same method applies as well for 4-regular plane graphs and their central circuit, see [9] for an exhaustive description.

\subsection{The case of $\left(\left\{v_{3}=4, v_{6}\right\}, 3\right)$-spheres}

All zigzags of $\left(\left\{v_{3}=4, v_{6}\right\}, 3\right)$-spheres are simple and the vector enumerating their lengths is of the form

$$
\left(4 s_{1}\right)^{m_{1}},\left(4 s_{2}\right)^{m_{2}},\left(4 s_{3}\right)^{m_{3}} \quad \text { with } \quad s_{i}, m_{i} \in \mathbb{N} \text { and } s_{i} m_{i}=\frac{n}{4} .
$$

This was first established in [17] but there is another way to establish it: Any $\left(\left\{v_{3}=\right.\right.$ $\left.\left.4, v_{6}\right\}, 3\right)$-sphere is obtained as a quotient of a $\left(\left\{v_{6}\right\}, 3\right)$-torus by a group of order 2 formed 

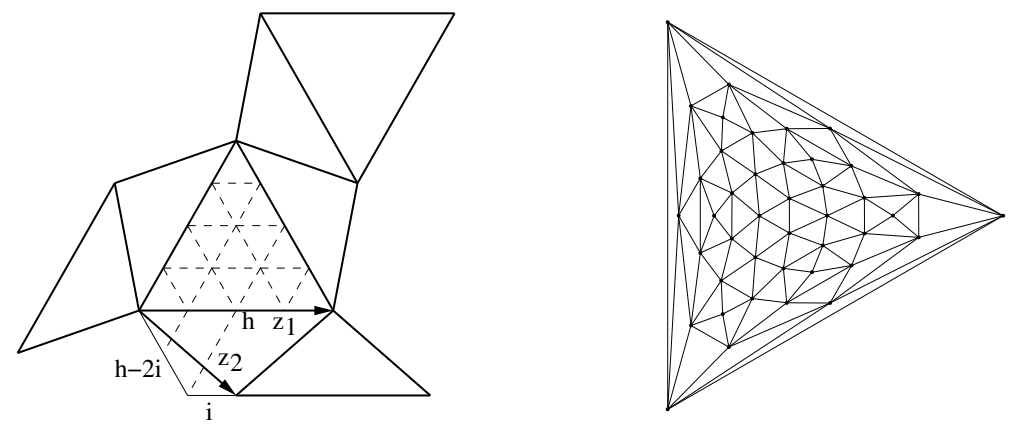

Figure 8: The parameter description of a family of $\left(\left\{v_{4}=6, v_{6}\right\}, 3\right)$-sphere with simple zigzags, $z_{1}=h \omega$ and $z_{2}=h-2 k+k \omega$ and the case $(h, k)=(4,1)$.

by inversion. The four vertices of degree 3 come from the four invariant vertices of the torus. All zigzags of a $\left(\left\{v_{6}\right\}, 3\right)$-torus are partitioned into three parallel classes that cover the vertex set. All the zigzags in a parallel class are of the same length and when passing to the quotient the parallel classes are preserved hence the above result is proved.

The same argument applies for $\left(\left\{v_{2}=4, v_{4}\right\}, 4\right)$-spheres and their central circuits [8].

\subsection{Other classes}

For other classes of maps with more parameters the structure is more complicated and it seems very difficult to obtain simple description of the zigzags of fullerenes. However, for $\left(\left\{v_{4}=6, v_{6}\right\}, 3\right)$-spheres we have a simple conjecture for the ones with simple zigzags:

Conjecture 4.1. All $\left(\left\{v_{4}=6, v_{6}\right\}, 3\right)$-spheres with only simple zigzags are:

- $G C_{k, k}($ Octahedron $)$ and

- the family of graphs with parameters $(m, k)$ with $n=4 h(2 h-3 k)$ triangles whose parameter description is given in Figure 8. The vector enumerating zigzag length is

$$
z=(6 h-6 k)^{3 h-3 k},(6 h)^{h-2 k},(12 h-18 k)^{k}
$$

They have symmetry $O_{h}$ if $k=0, D_{6 h}$ if $h=3 k$ and $D_{3 d}$ otherwise.

\section{References}

[1] G. Brinkmann, O. Delgado-Friedrichs, A. Dress and T. Harmuth, $\mathrm{CaGe}-\mathrm{a}$ virtual environment for studying some special classes of large molecules, MATCH-Commun. Math. Co. 36 (1997), 233-237.

[2] G. Brinkmann, T. Harmuth and O. Heidemeier, The construction of cubic and quartic planar maps with prescribed face degrees, Discrete Appl. Math. 128 (2003), 541-554.

[3] M. Conder and P. Dobcsányi, Determination of all regular maps of small genus, Combin. Theory Ser. $B 81$ (2001), 224-242.

[4] P. Deligne and G.D. Mostow, Monodromy of hypergeometric functions and nonlattice integral monodromy, Publ. Math. Inst. Hautes Études Sci. 63 (1986), 5-89. 
[5] M. Deza and M. Dutour, Zigzag Structure of Simple Two-faced Polyhedra, Comb. Probab. Comput. 14 (2005), 31-57.

[6] M. Deza and M. Dutour Sikirić, Zigzag and central circuit structure of $(\{1,2,3\}, 6)$-spheres, Taiwan. J. Math., to appear

[7] M. Deza, M. Dutour Sikirić and P. Fowler, The symmetries of cubic polyhedral graphs with face size no larger than 6, MATCH-Commun. Math. Co. 61 (2009), 589-602.

[8] M. Deza, M. Dutour and M.I. Shtogrin, 4-valent plane graphs with 2-, 3- and 4-gonal faces, "Advances in Algebra and Related Topics" (in memory of B.H. Neumann; Proceedings of ICM Satellite Conference on Algebra and Combinatorics, Hong Kong 2002), World Scientific Publ. Co. (2003), 73-97.

[9] M. Dutour and M. Deza, Goldberg-Coxeter construction for 3- and 4-valent plane graphs, Electron. J. Combin. 11 (2004), R\#20.

[10] M. Dutour Sikirić and M. Deza, 4-regular and self-dual analogs of fullerenes, Mathematics and Topology of Fullerenes, pp. 103-116 ed. by O. Ori, A. Graovac and F. Cataldo, Carbon materials, Chemistry and Physics, vol 4, Springer Verlag, 2011.

[11] M. Dutour Sikirić and P. Fowler, Cubic ramapolyhedra with face size no larger than 6, J. Math. Chem. 49 (2011), 843-858.

[12] P. W. Fowler, J.E. Cremona and J. I. Steer, Systematics of bonding in non-icosahedral carbon clusters, Theory Chem. Acta 73 (1988), 1-26.

[13] P. W. Fowler and D. E. Manolopoulos, An Atlas of Fullerenes, Clarendon Press, Oxford, 1995.

[14] M. Goldberg, A class of multisymmetric polyhedra, Tohoku Math. J. 43 (1937), 104-108.

[15] J. E. Graver, Encoding fullerenes and geodesic domes, SIAM J. Discrete Math. 17 (2004), 596614.

[16] J. E. Graver, Catalog of all fullerenes with ten or more symmetries, Graphs and discovery, 167-188 DIMACS Series Discrete Mathematics Theoretical Computer Science, 69, American Mathematical Society, 2005.

[17] B. Grünbaum and T. S. Motzkin, The number of hexagons and the simplicity of geodesics on certain polyhedra, Canadian J. Math. 15 (1963), 744-751.

[18] B. Grünbaum and J. Zaks, The existence of certain planar maps, Discrete Math. 10 (1974), 93-115.

[19] T. Harmuth, The construction of cubic maps on orientable surfaces, $\mathrm{PhD}$ thesis, Bielefeld, 2000.

[20] S. Jendrol', On face vectors of trivalent maps, Math. Slovaca 36 (1986), 367-386.

[21] P. E. John and H. Sachs, Spectra of toroidal graphs, Discrete Math. 309 (2009), 2663-2681.

[22] H. W. Kroto, J. R. Heath, R. F. Curl, R. E. Smalley, C60: Buckminsterfullerene, Nature 318 (1985), 162-163.

[23] G. D. Mostow, Generalized Picard lattices arising from half-integral conditions, Publ. Math. Inst. Hautes Études Sci. 63 (1986), 91-106.

[24] G. D. Mostow, Braids, hypergeometric functions, and lattices, B. Am. Math. Soc. 16 (1987), 225-246.

[25] S. Negami, Uniqueness and faithfulness of embedding of graphs into surfaces, Doctor thesis, Tokyo Institute of Technology, 1985.

[26] I. Rivin, Euclidean structures on simplicial surfaces and hyperbolic volume, Ann. Math. 139 (1994), 553-580. 
[27] C. H. Sah, A generalized leapfrog for fullerene structures, Fullerene Sci. Techn. 2 (1994), 445458.

[28] W. P. Thurston, Shapes of polyhedra and triangulations of the sphere, The Epstein birthday schrift, Geom. Topol. Monogr. 1 (1998), 511-549. 